\title{
MODELAGEM EM EXCEL PARA O PROBLEMA DE ALOCAÇÃO DE CUSTOS CONJUNTA UTILI- ZANDO O VALOR DE SHAPLEY
}

\author{
Giancarlo de França Aguiar ${ }^{1}$, BÁrbara C. X. C. Aguiar ${ }^{2}$, Volmir EUGÊNio Wilhelm ${ }^{2}$, PaUlo HenriQue \\ SIQUEIRA ${ }^{2}$
}

1. Departamento de Matemática, Instituto Federal do Paraná, Rua Joana Souza Gusso, 699, Boa Vista, Paraná, Curitiba.

E-mail: giancarlo.aguiareifpr.edu.br

\author{
2. Departamento de Expressão Gráfica, Universidade Federal do Paraná \\ Caixa Postal 19081, Centro Politécnico Edifício da Administração $4^{\circ}$ Andar Jardim das Américas \\ E-mail: babi.eg@ufpr.br, volmirw@gmail.com, paulohs@ufpr.br
}

\begin{abstract}
This paper explores a study of coalitions for decision making in division of joint costs. For this, it uses a methodology called Shapley Value (found in the literature as an application of game theory) to find a decision of cost sharing (either apportionment of profits). In this paper a numerical example is illustrated and its solution obtained from the modeling using the Microsoft Excel and solver optimization tool.
\end{abstract}

Keywords— Shapley Value, Cost Allocation Joint, Excel Modeling.

Resumo- Este trabalho explora um estudo de coalizões para a tomada de decisão na divisão de custos conjunta. Para isto, é utilizada a metodologia denominada Valor de Shapley (encontrada na literatura como uma aplicação da Teoria dos Jogos) para encontrar uma decisão de divisão dos custos (podendo ser rateio de lucros). Neste texto está ilustrado um exemplo numérico e sua solução obtida a partir da modelagem empregando a planilha eletrônica Microsoft Excel e a ferramenta de otimização solver.

Palavras-chave— Valor de Shapley, Alocação de Custos Conjunta, Modelagem em Excel.

\section{Introdução}

Neste trabalho iniciaremos o estudo de alocação de custos conjunta discutindo a seguinte situação fictícia: um casal pretende sair para um jantar a dois. Eles decidem que irão a uma praça de alimentação em um Shopping. Os dois optam por se deliciar comendo pizzas. O rapaz pretende comer sete pedaços de pizzas e a moça pretende comer três pedaços de pizzas. Eles podem individualmente comprar seus pedaços de pizzas, ou simplesmente comprar uma única pizza com dez pedaços e então realizar a partição.

Definiremos a partir de agora que comprar em conjunto representa uma coalizão. Desta maneira teremos os custos para a compra individual e os custos para a compra em conjunto (coalizão). Por hipótese o casal acaba percebendo, realizando contas elementares, que a compra conjunta vai reduzir os seus custos totais, e realizam a compra conjunta de uma única pizza. Ao chegarem ao caixa eles se deparam com a seguinte situação: como irão dividir os custos na compra desta pizza? A seguir ilustramos algumas possibilidades de pensamento.

1. Pagamento de meio-a-meio (metade da conta para ele e metade da conta para ela). As mulheres modernas não aceitam a submissão financeira dos homens e querem ratear a conta.

2. Pagamento proporcional ao consumo. $\mathrm{Ou}$ seja, ele pagaria $70 \%$ da conta e ela $30 \%$, já que ele vai comer sete pedaços e ela três pedaços de pizzas.

Caso eles tomem a decisão do meio-a-meio, a princípio o rapaz deve ficar mais satisfeito, pois ele irá comer muito mais pizzas e pagar a mesma parcela. Já a moça não deve apreciar muito a decisão, pois pagará metade da conta comendo muito menos. Caso o casal escolha o pagamento proporcional, ambos devem ficar satisfeitos (em teoria), pois irão pagar a proporção do que estão comendo. Essas situações na tomada de decisão podem ser chamadas de imputações, e serão discutidas com mais profundidade mais adiante.

Ao ir embora o rapaz começa a fazer uma nova reflexão. Acabei pagando por sete pedaços, contudo, minha colega comeu os três pedaços mais caros da pizza, e eu os sete pedaços mais baratos. Ou seja, mesmo realizando um rateio proporcional, o rapaz não saiu satisfeito com a imputação. É oportuno frisar que mediante qualquer tomada de decisão (mesmo parecendo justa), ainda assim alguém pode não sair totalmente satisfeito com a divisão. Este é um exemplo de situação fictícia para uma distribuição de custos conjunta. 
Podemos estender o problema de alocação de custos para o problema da distribuição de lucros conjunto. Vamos ilustrar um problema lúdico ilustrado no livro "O Homem que Calculava" de Julio Cesar de Mello e Souza, mais conhecido como Malba Tahan (pseudônimo) de 2001. É o problema dos 35 camelos. O livro conta a história de Bereniz, um jovem que desde cedo gostava de fazer contas. O jovem inicia contando pequenas quantidades e em pouco tempo já está contando o número de laranjas de um pé de laranjeira, o número de abelhas de um enxame, o número de ovelhas em um rebanho, entre outros.

O jovem Bereniz em uma de suas viagens encontra três irmãos discutindo sobre uma divisão de camelos que acabaram de herdar de seu pai falecido. A vontade do pai era que o filho mais velho ficasse com a metade de sua cáfila (conjunto de camelos), o filho do meio ficasse com um terço e o filho mais jovem ficasse com um nono. Os irmãos discutiam, pois o filho mais velho deveria receber metade de 35 camelos, ou seja, 17,5 camelos, o filho do meio deveria receber um terço de 35 camelos, ou seja, aproximadamente 11,66 camelos e o filho mais jovem um nono dos 35 camelos, ou seja, aproximadamente 3,88 camelos.

Acontece que nenhum dos irmãos aceitava receber uma quantidade inferior ao que havia sido estipulada na herança. Bereniz então se ofereceu para resolver o problema. Ele propôs dar o seu camelo aos três irmãos e então realizar a divisão, que ficou da seguinte forma: o irmão mais velho receberia metade de $35+$ 1 camelos, ou seja, 36/2 igual a 18 camelos, o irmão do meio receberia um terço de 36 camelos, ou seja, 12 camelos e o irmão mais jovem receberia um nono de 36 , ou seja, 4 camelos. Neste rateio todos os irmãos ficaram felizes com a divisão, pois receberam acima do esperado.

Somando o resultado das divisões: 18 camelos (irmão mais velho), com 12 camelos (irmão do meio), com 4 camelos (irmão mais jovem) obtém-se 34 camelos. Sobraram dois camelos que foram dados a Bereniz. Este é um exemplo lúdico de um rateio para um conjunto de três pessoas. Neste cenário é possível entender que o objetivo principal em problemas de alocação de custos é encontrarmos uma metodologia que deixe todos os jogadores mais satisfeitos quanto possível.

\section{Revisão de Literatura}

Este panorama (busca na redução de custos conjunta), aliado ao aumento crescente no número de privatizações em empresas tanto nacionais como internacionais, fez crescer a necessidade de se criarem modelos de distribuição de bens e serviços com qualidade e com custos minimizados. São fundamentais os modelos de distribuição de custos estruturados com alguma garantia ao consumidor, de que estão pagan- do pelo melhor serviço e com custos reduzidos (Moreira, Luna e Guedes, 2002).

Os problemas de alocação de custos vêm sendo fortemente discutidos em cursos de contabilidade (Beuren, 1993, Souza, Lisboa e Rocha, 2003, Harks e Miller, 2011). Os pesquisadores têm fomentado vários métodos (ou critérios de alocação) e empregado com sucesso em problemas reais. Novas metodologias ganharam força a partir do surgimento da teoria dos jogos cooperativos.

Littlechild e Thompson (1977) apud Figueiredo (1994) ilustram uma aplicação prática para a teoria dos jogos no rateio de custos no aeroporto de Birmingham (Inglaterra). A distribuição de custos tanto na construção, como na utilização das pistas de decolagem ocorre de maneira conjunta, e dessa forma, eles utilizaram a metodologia do nucléolo para encontrar o vetor de solução para aeronaves de diferentes tamanhos. Analisando as taxas pagas em 1968 e 1969 eles concluíram que as menores aeronaves subsidiavam as maiores aeronaves.

Moreira (2002) realizou um estudo da solução para o problema de alocação de custos (distribuição dos custos de aluguel das linhas de internet utilizadas pelos órgãos da prefeitura de Belo Horizonte) em uma empresa de informática e informação do município de Belo Horizonte, comparando a Teoria dos Jogos Cooperativos e uma heurística proposta (baseada na porcentagem de utilização da rede) pelos consumidores/órgãos da prefeitura). Seus resultados indicam que a heurística é apropriada ao solucionar o problema, contudo que não pode ser estendida para qualquer problema de alocação de custos.

Bezerra (2009) faz uma análise e caracterização de modelos de custos que utilizam o valor de Shapley para a alocação de custos entre departamentos. Este trabalho descreve as características de um modelo de alocação de custos baseado nos conceitos da teoria dos jogos cooperativos, e demostra que este modelo de distribuição de ganhos produz resultados que, para o tomador de decisão racional gera o melhor resultado de distribuição de custos.

Leite e Vieira (2010) realizou um estudo de caso em um trecho da bacia do rio Paraíba do Sul, utilizando como critérios para a alocação de custos por utilização de recursos hídricos, o volume de água captada e consumida. Eles utilizaram como modelo de simulação o valor de Shapley (pertencente à teoria dos jogos cooperativos) e concluíram que os resultados obtidos mostraram-se altamente dependentes dos critérios de alocação dos custos adotados.

\section{Metodologia}

A seguir serão definidos brevemente os conceitos de Núcleo, Nucléolo e Valor de Shapley, sendo o valor de Shapley o método escolhido para o processo de 
modelagem matemática e modelagem em Excel para este trabalho.

Imputação ou alocação é uma redistribuição exaustiva do valor de uma grande coalizão que atribui ou distribui para cada jogador no mínimo a quantidade que ele garantiria para si, se não entrasse para a grande coalizão. Nesse processo de barganha naturalmente uma imputação pode não satisfazer algum participante da coalizão.

Para Lamaire (1984), Yu, Benjaafar e Gerchak (2009), o Núcleo pode ser considerado o conjunto de todas as imputações não dominadas via qualquer coalizão, ou conjunto daquelas para as quais não existem objeções. Sua construção se dá eliminando do conjunto aquelas imputações que existem objeções.

Segundo Reinhardt (2004), o Nucléolo constitui um critério que seleciona imputações de forma a minimizar a máxima queixa ou reclamação que qualquer coalizão possa ter contra elas. Sua edificação está baseada na aplicação o Teorema Minimax de John Von Neumann.

David, Massol e Moisan (2005), abordam que o Valor de Shapley estabelece um critério que atribui a cada participante do jogo a sua contribuição marginal esperada. Assim o valor de Shapley pode ser considerado uma média de contribuição marginal do jogador i para a coalizão $S$, considerando que os jogadores formem essa coalizão em uma sequência aleatória. A Probabilidade de um jogador em particular participar em diversos momentos distintos, de coalizões com $\mathrm{S}$ participantes é definida pela equação 1 a seguir.

$$
(|S|-1) !(n-|S|) ! / n !
$$

Onde n representa o tamanho da amostra (número de elementos da coalizão). A contribuição marginal do jogador i em qualquer momento é definida pela equação 2.

$$
\mathrm{CM}_{\mathrm{i}}=[\mathrm{v}(\mathrm{S})-\mathrm{v}(\mathrm{S}-\{\mathrm{i}\})]
$$

Onde v(S) é o custo total da coalizão $S$ e v(S-\{i $\})$ é a diferença entre o custo total da coalizão $S$ e o custo da coalizão com exceção do jogador i. Desta forma o valor de Shapley (Média de contribuição marginal do jogador i para a coalizão $S$, em sequência aleatória), pode ser obtido utilizando a equação 3 abaixo.

$$
\phi_{\mathrm{i}}(\mathrm{v})=\sum_{\substack{\mathrm{S} \in \mathrm{N} \\ \mathrm{i} \in \mathrm{S}}} \frac{(|\mathrm{S}|-1) !((\mathrm{n}-|\mathrm{S}|) !}{\mathrm{n} !}[\mathrm{v}(\mathrm{S})-\mathrm{v}(\mathrm{S}-\{\mathrm{i}\})]
$$

A seguir está ilustrado um exemplo de problema de alocação de custos e sua resolução via Valor de Shapley. Uma empresa avalia se vale ou não a pena a centralização de serviços de TI. As seguintes situações são possíveis:
1. O Depto. A mantém a sua estrutura de TI por R\$ 5.000;

2. O Depto. B mantém a sua estrutura de TI por R\$ 3.000;

3. O Depto. C mantém a sua estrutura de TI por R\$ 5.000;

4. Se A e B mantém uma estrutura comum: $\mathrm{R} \$$ 6.000

5. Se A e C mantém uma estrutura comum: $\mathrm{R} \$$ 10.000 ;

6. Se B e C mantém uma estrutura comum: $\mathrm{R} \$$ 7.000

7. Se A, B e C mantém uma estrutura comum: $\mathrm{R} \$ 10.500$.

Encontre o valor de Shapley.

Neste problema o departamento A teria um custo de $\mathrm{R} \$$ 5.000,00, o departamento B um custo de R\$ $3.000,00$ e o departamento $\mathrm{C}$ um custo de $\mathrm{R} \$$ $5.000,00$ respectivamente se mantivessem suas estruturas de TI, contudo, se eles formassem uma coalizão seus custos individuais cairiam para 4.166,67, 1.666,67 e 4.666,67 adotando a metodologia valor de Shapley. Desta forma, todos os três departamentos possuem estímulos (redução no custo) para montar uma coalizão.

\section{Modelagem em Excel}

O modelo anterior pode ser resolvido utilizando algum software de programação linear, contudo neste momento, utilizaremos a ferramenta eletrônica Microsoft Excel para auxiliar no processo de modelagem e resolução (objetivo do trabalho). A figura 5 abaixo ilustra os dados inseridos na planilha eletrônica Microsoft Excel.

Identificar cada figura e tabela por um número sequencial.

Lembre sempre de colocar as unidades nos eixos dos gráficos e nas tabelas.

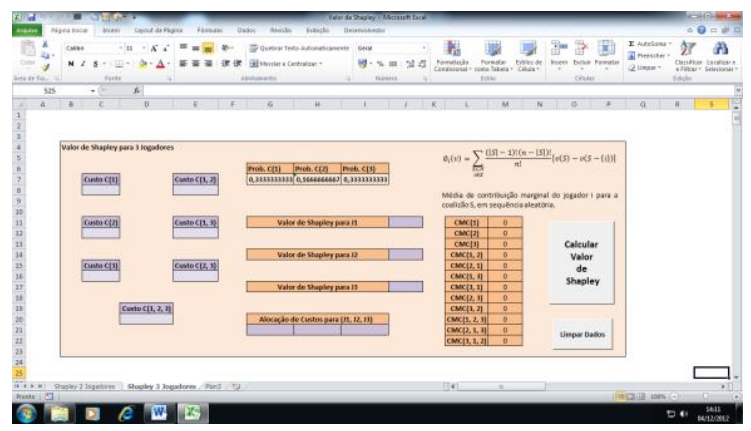

Figura 1. Interface de dados inseridos no Excel

As células $\mathrm{C} 8, \mathrm{C} 12$ e $\mathrm{C} 16$ receberam os valores de custos individuais dos departamentos A, B e C respectivamente sem nenhuma coalizão. A célula E8 recebeu o custo da coalizão dos departamentos $\mathrm{A}$ e $\mathrm{B}$, a célula E12 dos departamentos A e C, a célula 
E16 dos departamentos B e C, e a célula D20 recebeu os custos da coalizão dos três departamentos A, B e C. As células $\mathrm{G} 7, \mathrm{H} 7$ e $\mathrm{I} 7$ receberam as probabilidades de entrada dos departamentos (A ou B ou C) na coalizão em momentos diferentes, ou seja, em primeiro, segundo e terceiro lugares, que são respectivamente as probabilidades 1/3,1/6 e 1/3.

A célula $\mathrm{J} 11$ recebe a função $(=\mathrm{G} 7 * \mathrm{M} 11+\mathrm{H} 7 * \mathrm{M} 14+\mathrm{H} 7 * \mathrm{M} 16+\mathrm{I} 7 * \mathrm{M} 20)$ que representa o valor de Shapley para o jogador 1 (departamento A), sendo este resultado o somatório dos produtos de sua probabilidade de entrada na coalizão, pelo seu custo marginal, a célula $\mathrm{J} 14$ recebe a função $(=\mathrm{G} 7 * \mathrm{M} 12+\mathrm{H} 7 * \mathrm{M} 15+\mathrm{H} 7 * \mathrm{M} 18+\mathrm{I} 7 * \mathrm{M} 21)$ que representa o valor de Shapley para o jogador 2 (departamento B), e a célula $\mathrm{J} 17$ recebe a função $(=\mathrm{G} 7 * \mathrm{M} 13+\mathrm{H} 7 * \mathrm{M} 17+\mathrm{H} 7 * \mathrm{M} 19+\mathrm{I} 7 * \mathrm{M} 22)$ que representa o valor de Shapley para o jogador 3 (departamento $\mathrm{C}$ ).

As células M11 (=C8-0), M12 (=C12-0), M13 (=C16-0), M14 (=E8-C12), M15 (=E8-C8), M16 (=E12-C16), M17 (=E12-C8), M18 (=E16-C16), M19 (=E16-C12), M20 (=D20-E16), M21 (=D20E12) e M22 (=D20-E8) representam as contribuições marginais dos três departamentos segundo a ordem de entrada ou não na coalizão. As células G21, H21 e I21 recebem os resultados J11, J14 e J17 respectivamente, que é o valor de Shapley para cada jogador. Os botões "Calcular o Valor de Shapley" e "Limpar Dados" foram criados usando a ferramenta desenvolvedor com controles de formulário e gravando macros no Excel. A figura 6 a seguir ilustra os resultados do problema utilizando a planilha eletrônica Microsoft Excel.

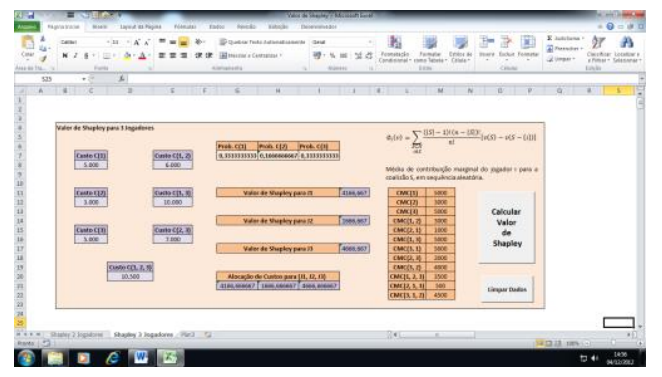

Figura 2. Resultados com a Planilha Eletrônica Microsoft Excel

Os resultados (alocação de custos para o jogador 1 igual a $\mathrm{R} \$ 4.166,66$, para o jogador 2 igual a $\mathrm{R} \$$ $1.666,66$ e para o jogador 3 igual a $\mathrm{R} \$ 4.666,66)$ indicam a coalizão entre os departamentos como a melhor solução para cada jogador.

\section{Considerações Finais}

Por meio de um exemplo simplificado (o problema dos 35 camelos), foi ilustrado neste trabalho o significado descritivo e quantitativo para o problema de alocação de custos conjunta.
Foi realizada uma síntese de quatro trabalhos científicos (que constam na base de dados SciELO Brasil) que discursam sobre o problema de alocação de custos em Teoria dos Jogos, objetivando a construção de um referencial teórico para o leitor.

Este texto discutiu uma metodologia (o Valor de Shapley) para o problema de alocação de custos conjunta que resulta em bons resultados para os jogadores (tomadores de decisão na Teoria dos Jogos).

E por fim, foi instruído como encontrar o Valor de Shapley para um conjunto de jogadores, utilizando para isto, a modelagem de um problema através da planilha eletrônica Microsoft Excel.

\section{Referências Bibliográficas}

BEUREN, Ilze Maria. "Evolução Histórica da Contabilidade de Custos". Contabilidade Vista \& Ver., vol. 5, n. 1, pág. 61-66, Belo Horizonte, 1993.

BEZERRA, F. A., GRANDE, J. F., SILVA, A. J. Análise e Caracterização de Modelos de Custos que Utilizam o Valor de Shapley para Alocação de Custos entre Departamentos. Gestão \& Produção, vol. 16, n. 1, São Carlos, 2009.

DAVID, Laurent; MASSOL, Olivier; MOISAN, Anthony. "Shapley Value as a Joint Cost Allocation Mechanism. Is the Story Definitely Over?". CiteSeerX Scientific Literature Digital Library and Search Engine, Gas de France, July 2005.

FIGUEIREDO, R. S. Teoria dos Jogos: Conceitos, Formalização Matemática e Aplicação à Distribuição de Custo Conjunto. Gestão \& Produção, vol. 1, n. 3, São Carlos, 1994.

HARKS, Tobias; MILLER, Konstantin. "The Worst Case Efficiency of Cost Sharing Methods in Resource Allocation Games". Operations Research, vol. 59, n. 6, pág. 1491-1503, December 2011. DOI: 10.1287/opre.1110.0979

LAMAIRE, Jean. "An Application of Game Theory: Cost Allocation". Astin Bulletin, vol. 14, n. 1, Université Libre de Bruxelles, 1984.

LEITE, G. B., VIEIRA, W. C. Proposta Metodológica de Cobrança pelo uso dos Recursos Hídricos Usando o Valor de Shapley: uma Aplicação à Bacia do Rio Paraíba do Sul. Estudos Econômicos, vol. 40, n. 3, São Paulo, 2010.

LITTECHILD, S. C., THOMPSON, G. F. Aircraft Landing Fees: A Game Theory Approach. Bell Journal of Economics, vol. 8, n. 1, spring 1977.

MOREIRA, R. C.; LUNA, H. P. L.; GUEDES, P. G. S. Um estudo comparativo entre a teoria dos jogos cooperativos e uma heurística aplicados a um problema real de alocação de custos. Pesquisa Operacional vol. 22, n. 1, Rio de Janeiro, 2002. 
REINHARDT, Gilles. "Allocating the Cost of Congestion With the Nucleolus". Computational Economics, vol. 24, issue 1, pág. 21-33, August 2004.

SOUZA, Marcos A.; LISBOA, Lázaro, P.; ROCHA, Welington. "Práticas de Contabilidade Gerencial Adotadas por Subsidiárias Brasileiras de Empresas Multinacionais". Revista Contabilidade \& Finanças, vol. 14, n. 32, São Paulo, Agosto 2003.

TAHAN, M.; O Homem que Calculava. Editora Record, $55^{\circ}$ ed., 2001.

YU, Yimin; BENJAAFAR, Saif; GERCHAK, Yigal. "Capacity Sharing and Cost Allocation Among Independent Firms in the Presence of Cosgestion". University of Minnesota Minneapolis. Fonte: http://isye.umn.edu/faculty/pdf/ybg-04-05-09.pdf , 2009. 